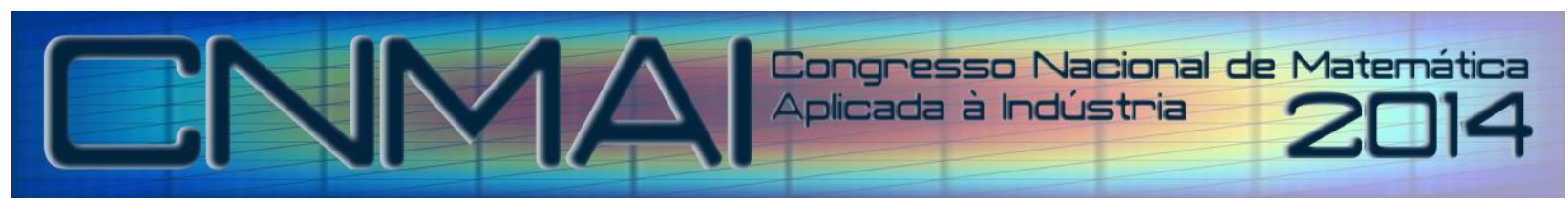

18 a 21 de novembro de 2014, Caldas Novas - Goiás

\title{
AVALIAÇÃO DAS GEOMETRIAS DAS CURVAS UTILIZADAS PARA REPRESENTAR AS ISOTERMAS DAS SUPERFÍCIES DAS CHAPAS SOLDADAS
}

\author{
Dario Magno Batista Ferreira, dariomagno@usp.br ${ }^{1}$ \\ Antonio do Nascimento Silva Alves, ansa@ usp.br ${ }^{1}$ \\ Rubelmar Maia de Azevedo Cruz Neto, rubelmar.neto@usp.br ${ }^{1}$ \\ Sérgio Duarte Brandi, sbrandi@usp.br ${ }^{1}$ \\ ${ }^{1}$ USP - Dep. de Eng. Metalúrgica e de Materiais. Av. Professor Mello Moraes, 2463, CEP 05508-030, São Paulo - SP
}

Resumo: A transferência de calor na soldagem é um fenômeno regido pela Equação de Fourier, e ocorre entre a poça de fusão, sua vizinhança e as condições de contorno envolvidas. Caracteriza-se pelos elevados gradientes térmicos e elevadas taxas de aquecimento e de resfriamento, que são observados pelas distribuições das isotermas e pelos ciclos térmicos obtidos. As isotermas são de suma importância, pois descrevem graficamente os gradientes térmicos da transferência de calor em todas as direções. Uma isoterma de particular interesse é a da linha de fusão, pois delimita o contorno da poça de fusão, ou seja, delimita as regiões entre o metal líquido e o metal sólido. Os modelos mais comumente utilizados na literatura permitem descrever os gradientes de temperatura, em regime transiente e em regime pseudoestacionário, como são os casos dos modelos desenvolvidos por Rosenthal para a transferência de calor por condução. As equações que são soluções destes modelos combinam funções transcendentais e funções especiais do tipo Bessel modificada, dependendo do modelo em estudo, em que se combinam parâmetros operacionais de soldagem, propriedades físicas e geométricas da chapa a ser soldada. A obtenção de isotermas é conseguida pelo cálculo complexo de funções inversas das soluções ou por um processo de cálculo de raízes de funções, por exemplo. Este último mecanismo será adotado neste trabalho. Entretanto, em simulações numéricas de soldagem, a isoterma representada por uma dupla elipsoide tem sido empregada para simplifica-la. Isto traz a vantagem do ganho computacional em função de sua simplicidade em relação ao modelo de Rosenthal. Neste sentido, propõe-se avaliar a extensão deste ganho ao verificar se a simplificação em questão é viável para diferentes materiais e condições operacionais de soldagem. Esta análise foi feita ao comparar os desvios das geometrias dos elipsoides em relação às soluções analíticas. A difusividade térmica e a velocidade de soldagem foram determinantes na quantificação do erro.

Palavras-chave: soldagem, simulação, isoterma, ciclo térmico, Elipsoide

\section{INTRODUÇÃO}

A soldagem a arco elétrico é um processo de união de peças muito utilizado na construção de estruturas metálicas na indústria, de um modo geral. $\mathrm{O}$ aporte energético imposto pelo processo promove a fusão na região da junta, na presença ou não de um metal de adição, de tal modo que ocorrem transformações metalúrgicas não só na mudança do estado líquido para o sólido na poça de fusão, como também, na região não fundida, adjacente a poça de fusão, conhecida como zona afetada pelo calor (ZAC). Nestas regiões, os gradientes térmicos de aquecimento e de resfriamento são bastante elevados, em função da transferência de energia térmica para a peça, a partir de uma fonte de calor bastante localizada. O estudo e o domínio destas transformações são de suma importância para previsão e controle das microestruturas resultantes, e, consequentemente, das propriedades requeridas para a junta soldada, segundo Wainer et al. (1992). Goldak, et al. (1984) afirmam que, por estas razões, diversos pesquisadores têm trabalhado para obtenção de um bom modelo da fonte de calor para análise de ciclos térmicos de soldagem, embora os modelos desenvolvidos por Rosenthal (1941) sejam ainda bastante referenciados. Neste trabalho, far-se-á uma investigação a respeito da geometria da poça de fusão em função de três modelos matemáticos da fonte calor, utilizados para descrever os 
fenômenos térmicos que ocorrem na ZAC, a partir da linha de fusão. Estes modelos são aplicados para os cálculos de ciclos térmicos na soldagem.

\section{MODELAGEM MATEMÁTICA}

O fenômeno da transferência de calor na soldagem é regido pela equação de Fourrier, conforme a Eq. (1), onde $T$ é a temperatura $\left({ }^{\circ} \mathrm{C}\right), t$ o tempo (s) e $\alpha$ a difusividade térmica $\left(\mathrm{mm}^{2} / \mathrm{s}\right)$ e $x, y$ e $z$ são os eixos do sistema de coordenadas.

$$
\frac{\partial^{2} T}{\partial x^{2}}+\frac{\partial^{2} T}{\partial y^{2}}+\frac{\partial^{2} T}{\partial z^{2}}=\frac{1}{\alpha} \frac{\partial T}{\partial t}
$$

A partir desta equação, modelos têm sido propostos considerando materiais isotrópicos, fontes de calor puntiforme, linear, soldagem de cordão sobre chapa, propriedades físicas independentes da temperatura, entre outras simplificações, de tal modo a obter uma solução analítica. Por estas razões, valores calculados da distribuição de temperaturas desviamse um pouco dos valores experimentais. As equações obtidas dão um bom entendimento do comportamento térmico da soldagem, conforme Lancaster (1999).

Entretanto, em muitas aplicações de soldagem, pesquisadores formulam modelos alternativos para o perfil das isotermas, como é o caso de Goldak et al. (1984), cujos trabalhos tem sido bastante referenciados em simulações numéricas de soldagem. Ele aproximou sua fonte de calor (isoterma) por uma dupla de elipsoides, com uma distribuição gaussiana da densidade de potência, limitada pelos semi-eixos ( $a, b, c_{1}$ e $\left.c_{2}\right)$ dos elipsoides, conforme a Fig. 1, ao simular a distribuição de temperaturas na soldagem através do Método de Elementos Finitos (MEF).

Bate et al. (2009) utilizaram o conceito de duplo elipsóide desenvolvido por Goldak et al. (1984), cujo código está presente em um software comercial de Elementos Finitos, para analisar tensões residuais de soldagem, onde as propriedades físicas do material são dependentes da temperatura e ainda foram considerados os efeitos da transferência de calor por convecção e por radiação, na poça de fusão. Guimarães et al. ( 2012) também fizeram uso do duplo elipsóide em suas simulações de um processo de soldagem de uma chapa de aço inoxidável AISI 304 com $3 \mathrm{~mm}$ de espessura, pelo processo GTAW, em que simulam os ciclos térmicos.

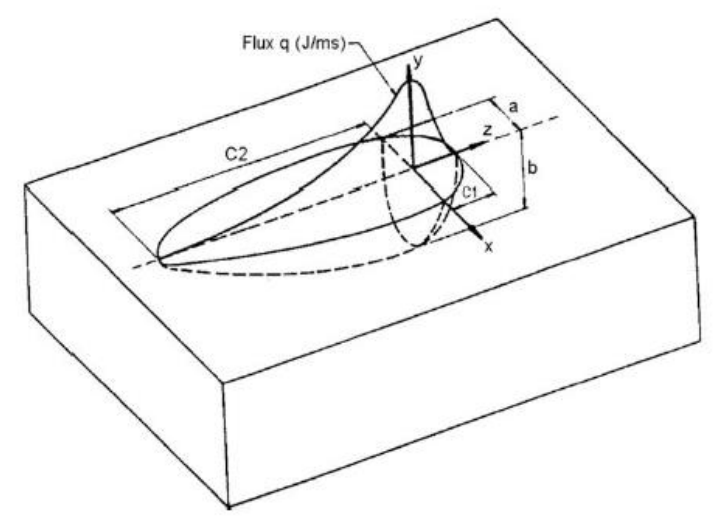

Figura 1 - Fonte calor duplo elipsóide e distribuição de potência, extraído de Goldak et al. (1984).

Eagar e Tsai (1983) utilizaram uma fonte circular com distribuição gaussiana da energia e comparou os resultados teóricos com experimentais através dos processos GTAW e GMAW em aço carbono e outros metais ou ligas.

\section{1 - Soluções analíticas da transferência de calor na soldagem}

Como citado anteriormente, os modelos analíticos desenvolvidos por Rosenthal, para fontes de calor móveis no final da década de 1930 (Rosenthal, 1935; Boulton e Martin, 1937; Rosenthal e Schmerber, 1938), são ainda os mais utilizados para o cálculo da história térmica de soldagem, relata Goldak et al. (1984).

Sendo assim, o modelo denominado chapa grossa, representado pela Fig. 2, admite uma fonte de calor puntiforme que se move com potência $q_{0}(\mathrm{~W})$ e velocidade $v(\mathrm{~mm} / \mathrm{s})$ constantes, ao longo de uma chapa semi-infinita. Assume como condição de contorno o fluxo de calor nulo na face inferior, e não leva em conta sua espessura. A solução da Eq. (1) para este caso, em regime pseudoestacionário, é expressa pelas Eq. (2) seguinte:

$$
\begin{gathered}
T-T_{0}=\frac{q_{0}}{2 \pi \lambda}\left(\frac{1}{R}\right) \exp \left[-\frac{v}{2 \alpha}(R+x)\right] \\
R=\sqrt{x^{2}+y^{2}+z^{2}}
\end{gathered}
$$


Os valores da condutividade térmica $\lambda\left(\mathrm{W} / \mathrm{mm}^{\circ} \mathrm{C}\right)$ e de outras propriedades térmicas são assumidos independentes da temperatura. Neste caso, todas as isotermas deste modelo admitem fluxo de calor nas três direções para dentro da chapa. As isotermas vistas a partir do plano $x-y$ assumem forma de semicircunferências.

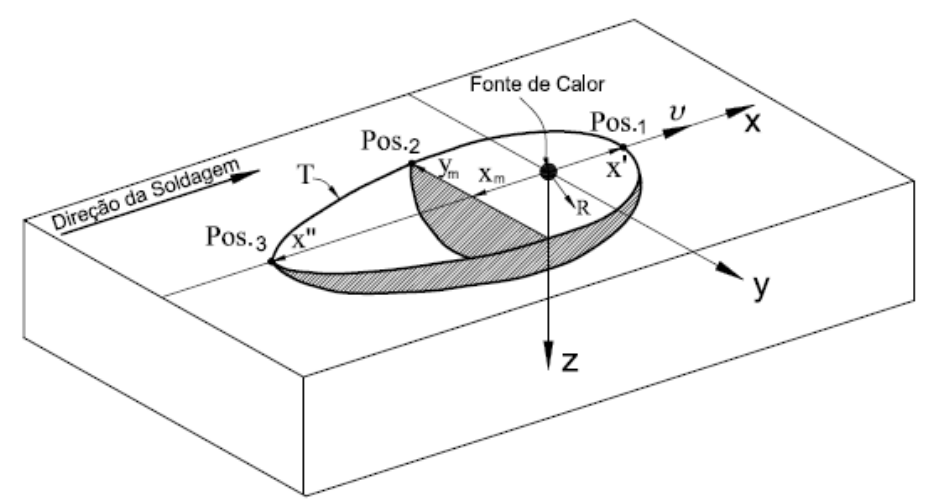

Figura 2. Isoterma tridimensional em uma chapa grossa.

Adicionalmente, o modelo denominado chapa média, desenvolvido por Rosenthal (1941), adota uma chapa semiinfinita com espessura $d(\mathrm{~mm})$ e as condições de contorno definidas por:

$$
\left\{\begin{array}{l}
\frac{\partial T}{\partial z}=0, \quad z=0 \\
\frac{\partial T}{\partial z}=0, \quad z=d
\end{array}\right.
$$

Isto implica que não há perda de calor por radiação e convecção pelas superfícies superior e inferior da chapa. Uma fonte puntiforme move-se em linha reta com velocidade constante $v(\mathrm{~mm} / \mathrm{s})$. Em Grong (1997), fontes imaginárias são dispostas a uma distância $2 i d(\mathrm{~mm})$ em relação à posição da fonte real, conforme mostra a Fig. 3, de tal modo que, uma fonte de calor anula o efeito da outra simetricamente oposta.

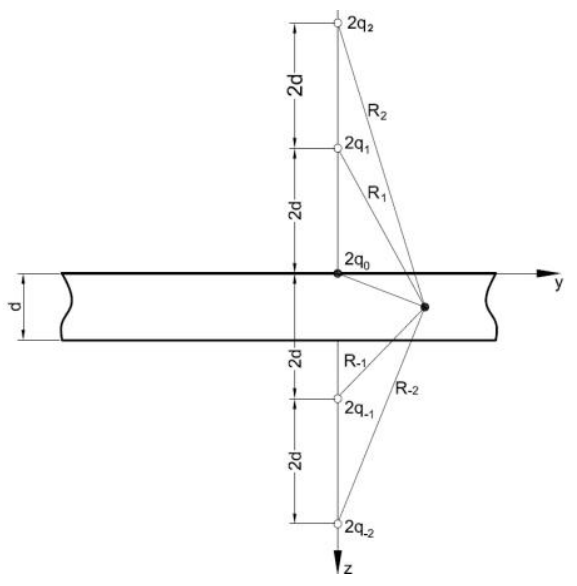

Figura 3. Disposição das fontes real e imaginárias sobre uma chapa média, Grong (1997).

A solução da Eq. (1) em função das condições de contorno da Eq. (3), em regime pseudoestacionário, é definida pela Eq. (4). $R_{i}(\mathrm{~mm})$ é o vetor raio que mede a distância entre a fonte imaginária $i$ ao ponto onde se deseja medir a temperatura.

$$
\begin{gathered}
T-T_{0}=\frac{q_{0}}{2 \pi \lambda} \exp \left(-\frac{v x}{2 \alpha}\right) \cdot\left[\sum_{i=-\infty}^{i=+\infty} \frac{1}{R_{i}} \exp \left(-\frac{v}{2 \alpha} R_{i}\right)\right] \\
R_{i}=\sqrt{x^{2}+y^{2}+(z-2 i d)^{2}}
\end{gathered}
$$

A Figura 4 exibe o perfil das isotermas em uma chapa média, considerando todas as vistas. As isotermas deste modelo assumem fluxo de calor em todas as direções, nas proximidades da linha de fusão. Assumem ainda fluxos de 
calor bi e unidimensional, à medida que se afastam da fonte de calor e da face superior, dependendo das condições operacionais, propriedades térmicas e dimensões da chapa.

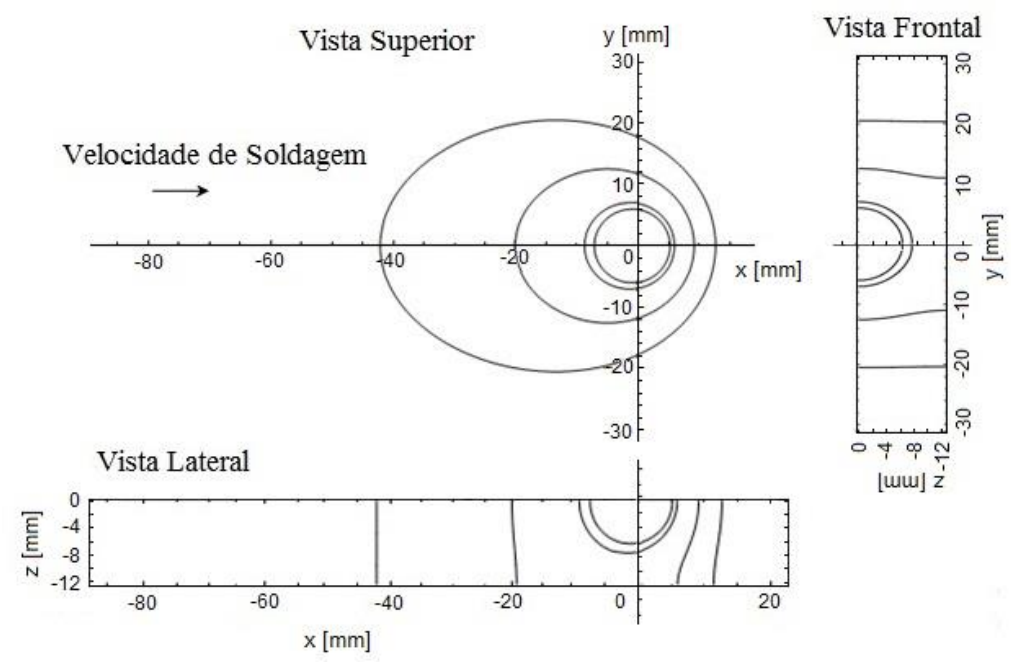

Figura 4. Isotermas em uma chapa média

A partir das Eqs. (2a) e (4a), pode-se deduzir que o modelo de chapa média é o caso geral do modelo de chapa grossa. Alternativamente, o modelo denominado chapa fina de Rosenthal (1941) admite uma chapa grande de espessura finita $d$, sendo soldada em apenas um passe por uma fonte de calor linear, alcançando de uma só vez toda sua espessura. A Eq. (5a) e a Eq. (5b) definem a solução para este modelo, onde $\mathrm{K}_{0}$ é a função de Bessel de segundo tipo e ordem zero.

$$
\begin{gathered}
T-T_{0}=\left(q_{0} / 2 \pi \lambda d\right) \exp (-v x / 2 \alpha) K_{0}(-v r / 2 \alpha) \\
r=\sqrt{x^{2}+y^{2}}
\end{gathered}
$$

Este modelo caracteriza-se por não possuir gradiente de temperatura ao longo de sua espessura, e seu fluxo de calor será bidimensional ou unidimensional, no plano $x-y$. A Figura 5 exibe um esboço da isoterma de uma chapa fina.

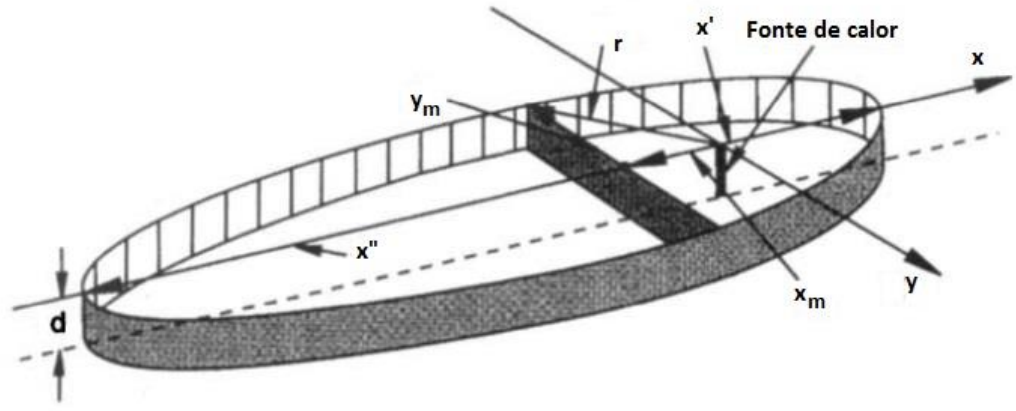

Figura 5. Representação gráfica do modelo de chapa fina, adaptado de Grong (1997)

De um modo geral, as isotermas podem ser representadas pelo esboço da isoterma sobre a face superior da Fig. 2, onde alguns pontos estão caracterizados: $x^{\prime}$ e $x^{\prime \prime}$ caracterizam seus extremos ao longo do eixo x, assim como $y_{m}$ caracteriza metade de sua largura. No caso em que a isoterma T é o ponto de fusão do metal, então $y_{m}$ será a metade da largura do cordão de solda.

As geometrias das isotermas destes modelos são influenciadas pela potência líquida de entrada, $q_{0}(\mathrm{~W})$, pela velocidade de soldagem e pela difusividade térmica do material a ser soldado. Ou seja, um aumento na potência líquida e/ou na velocidade de soldagem tende a alongar a geometria da isoterma, impondo-lhe um formato de lágrima. Por outro lado, uma alta difusividade térmica contribui para a variação de um perfil que passa pelo circular ao elíptico, quando se aumentam a velocidade de soldagem e/ou a potência líquida de entrada, segundo Grong (1997).

Grong (1997) afirma ainda que o modelo de chapa média é o mais adequado para situações práticas de soldagem para prever a distribuição de temperaturas de pico e levantamento de ciclos térmicos dentro da região da ZAC, para diversas condições operacionais, desde a soldagem de alumínio (alta difusividade térmica) até a soldagem de aço (baixa difusividade térmica).

\section{2 - Definição dos parâmetros da dupla elipse}


Este trabalho fará uma investigação a respeito da adequação do duplo elipsóide em relação aos modelos de chapa grossa, chapa média e chapa fina propostos por Rosenthal (1941). Tendo em vista que o elipsóide é a forma geométrica gerada a partir da rotação de uma elipse, a análise em questão será realizada considerando uma dupla de elipses sobre a face superior da chapa, ao invés do duplo elipsóide.

Assim, considerando a equação parametrizada da elipse, definida pela Eq. (6), $a$ e $b$ são os seus semi-eixos (nas direções $x$ e $y$, respectivamente), enquanto que $h$ e $k$ são as coordenadas do seu centro. Assim,

$$
\left\{\begin{array}{l}
x=h+a \cos (t) \\
y=k+b \operatorname{sen}(t)
\end{array}\right.
$$

Uma vez definida a geometria da fonte calor, resta então definir seus parâmetros, de tal modo a poder comparar com as geometrias das isotermas das soluções analíticas. Neste caso, a melhor configuração é aquela em que a elipse da esquerda compreenderá a parte da isoterma da Fig. 2, desde a posição 3 até a posição 2, enquanto a elipse da direita compreenderá o intervalo desde a posição 1 até a posição 2. Assim, a dupla elipse terá tamanho e forma mais próximas das isotermas.

Deste modo,

- o ponto do centro será comum as duas elipses, cujas coordenadas são $h=x_{m}$ e $k=0$;

- o semi-eixo vertical também será comum às duas elipses, assim, $b=y_{m}$;

- $\quad$ o semi-eixo horizontal da elipse da esquerda será $a_{e s q}=x^{\prime \prime}-x_{m}$;

- $\quad$ o semi-eixo horizontal da elipse da direita é definido por $a_{d i r}=x_{m}+x^{\prime}$.

Para calcular os pontos acima descritos, foi necessário utilizar as equações dos modelos analíticos apresentados anteriormente em variáveis adimensionais, onde a distribuição de temperaturas e os pontos em questão estão caracterizados por equações próprias. Entretanto, como se verá adiante, a maioria destas equações são funções transcendentais. Ou seja, torna-se necessário a utilização de código computacional para que elas sejam avaliadas. O método numérico utilizado neste trabalho foi o do cálculo de raízes de equações. Cada isoterma foi plotada pela metade com 401 pontos. Cada quadrante de elipse foi plotado por 201 pontos. Para cada ponto de isoterma calculado, o código computacional desenvolvido no software Wolfram Mathematica ${ }^{\circledR}$ realizou uma série de iterações para encontrar a raiz da função em estudo.

\section{3 - Estudo do erro entre a dupla elipse e os modelos desenvolvidos por Rosenthal}

A Figura 6 mostra os perfis da isoterma de $723^{\circ} \mathrm{C}$ de uma dada condição de soldagem, pelo modelo de Chapa grossa e a dupla elipse correspondente, tendo em vista o entendimento do comportamento da curva de erro entre a solução analítica e a solução alternativa (dupla elipse). A princípio, foi analisada a diferença entre as extensões das curvas $(\Delta y)$ em função da abcissa (Fig. 6b), para a temperatura em questão. Os estudos de caso seguintes analisarão o erro máximo para temperaturas de interesse para cada condição de soldagem para e modelo de chapa.

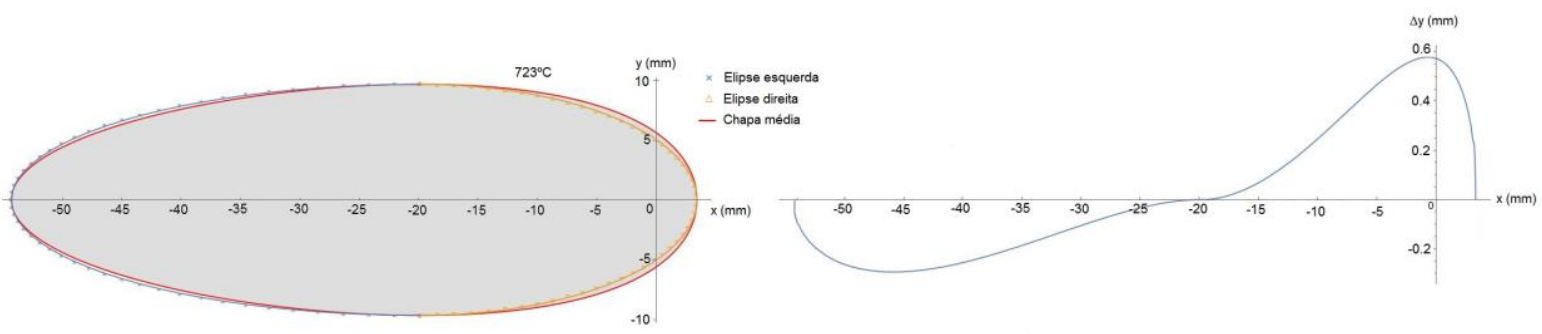

(a)

(b)

Figura 6. Perfis da isoterma de $723^{\circ} \mathrm{C}$ e da dupla de elipses, $v=8 \mathrm{~mm} / \mathrm{s}$

\section{a) Estudo do erro em relação à Chapa grossa, em variáveis adimensionais}

A equação da distribuição de temperatura para o modelo de chapa grossa, em variáveis adimensionais e em regime pseudoestacionário (Grong, 1997), é definido pela Eq. (7), onde $\sigma_{3}$ é o vetor raio adimensional, $\theta$ a temperatura adimensional e $n_{3}$ o parâmetro operacional, definidos pela Eq. (8). O sistema de coordenadas adimensional é definido pelas variáveis $\xi$, $\psi$ e $\zeta$, que fazem analogia com os eixos $x, y$ e $z$, respectivamente.

$$
\begin{gathered}
\theta / n_{3}=\exp \left(\sigma_{3}-\xi\right) / \sigma_{3} \\
\theta=\left(T-T_{0}\right) / T_{c}-T_{0}
\end{gathered}
$$




$$
\begin{gathered}
\sigma_{3}=(v R / 2 \alpha)=\sqrt{\xi^{2}+\psi^{2}+\zeta^{2}} \\
n_{3}=q_{0} v / 4 \pi \alpha^{2} \rho c\left(T_{c}-T_{0}\right)=q_{0} v / 4 \pi \alpha^{2}\left(H_{c}-H_{0}\right)
\end{gathered}
$$

Os pontos $\xi^{\prime}, \xi^{\prime \prime}, \xi_{m}$ e $\psi_{m}$ definidos na Eq. (9) representam, respectivamente, os pontos x', $x^{\prime \prime}, x_{m}$, e $y_{m}$.

$$
\begin{gathered}
\xi^{\prime}=\ln \left(n_{3} / \xi^{\prime} \theta\right) / 2 \\
\xi^{\prime \prime}=-n_{3} / \theta \\
\xi_{m}=-\left(\sigma_{3 m}\right)^{2} /\left(\sigma_{3 m}+1\right) \\
\psi_{m}=\zeta_{m}=\sqrt{\left(\sigma_{3 m}\right)^{2}-\left(\xi_{m}\right)^{2}}
\end{gathered}
$$

Estudo de caso 1: Soldagem de aço baixa ligam, processo GMAW: corrente $(I)=300 \mathrm{~A}$, tensão $(U)=25 \mathrm{~V}, v_{l}=$ $4 \mathrm{~mm} / \mathrm{s}$ e $v_{2}=8 \mathrm{~mm} / \mathrm{s}$. Dados obtidos do exemplo 1.12, Grong (1997). A eficiência do processo $(\eta)$ adotada será igual a 0,8 .
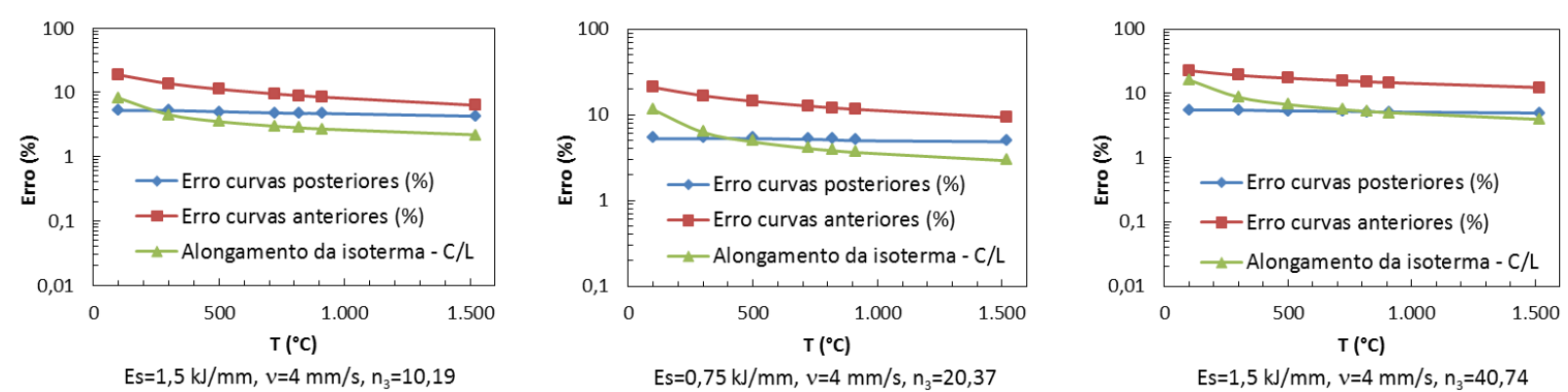

Figura 7. Curvas de erro para aço baixa liga, condição de transferência de calor para chapa grossa.

A Figura 7 exibe o comportamento das curvas de erro para três condições de soldagem de aço baixa liga $(\alpha=5$ $\mathrm{mm}^{2} / \mathrm{s}$ ). Nela estão considerados os erros em relação às curvas posteriores e anteriores à fonte de calor e a razão entre o comprimento da isoterma $(C)$ e sua largura $(L)$ para diversas temperaturas. Algumas temperaturas foram escolhidas porque correspondem a temperaturas em que ocorrem transformações metalúrgicas, ou seja, $1520^{\circ} \mathrm{C}$ corresponde à temperatura de fusão, $912^{\circ} \mathrm{C}$ é a temperatura $\mathrm{AC}_{3}$ (menor temperatura em que um aço baixo carbono é todo austenítico) e $723^{\circ} \mathrm{C}$ é a temperatura na qual ocorre a transformação total da austenita em ferrita e perlita, para aços hipoeutetóides ASM Handbook (1991). As demais temperaturas foram escolhidas para se verificar a extensão dos erros estudados.

Observa-se um erro entre $4 \%$ e $5 \%$ na elipse posterior à fonte de calor, para as três condições de soldagem impostas. Entretanto, ele é superior na elipse anterior e atinge valores superiores a $20 \%$. O erro cresce com a diminuição da temperatura e com o aumento do fator operacional $\mathrm{n}_{3}$, conforme exibe a Fig. (7).

Estudo de caso 2: Soldagem de alumínio: processo GMAW, $\eta=0,8, I=260 \mathrm{~A}, U=25 \mathrm{~V}, v_{1}=3 \mathrm{~mm} / \mathrm{s}$ e $v_{2}=6 \mathrm{~mm} / \mathrm{s}$. Dados obtidos do exemplo 1.12 de Grong (1997). Os resultados estão sumarizados na Fig. (8).

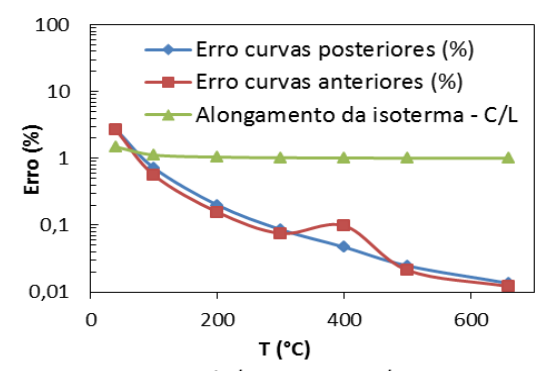

$E s=1,73 \mathrm{~kJ} / \mathrm{mm}, v=3 \mathrm{~mm} / \mathrm{s}, \mathrm{n}_{3}=0,10$

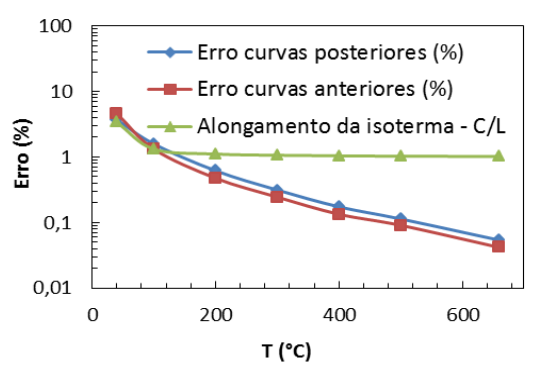

$E s=0,87 \mathrm{~kJ} / \mathrm{mm}, v=6 \mathrm{~mm} / \mathrm{s}, \mathrm{n}_{3}=0,20$

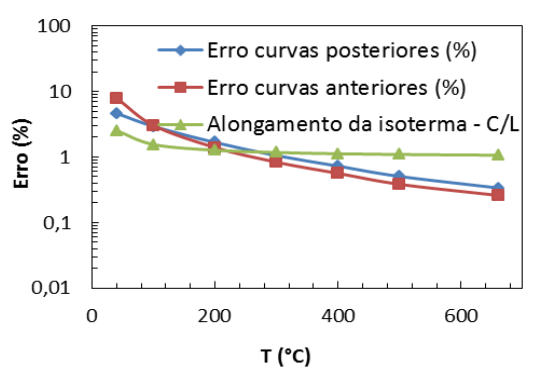

$E s=0,71 \mathrm{~kJ} / \mathrm{mm}, v=10,16 \mathrm{~mm} / \mathrm{s}, \mathrm{n}_{3}=0,47$

Figura 8. Curvas de erro para alumínio, para a condição de transferência de calor para chapa grossa.

A terceira condição de soldagem foi retirada da American Welding Society ( 2001) para uma chapa de alumínio com 19,05mm de espessura, processo de soldagem GMAW com argônio como gás de proteção. Os parâmetros de soldagem foram 340 A e $26 \mathrm{~V}$. Em todos os casos, houve aumento do erro, sendo mais significativo para a terceira condição, onde o erro máximo foi de $7,9 \%$, na temperatura de $40^{\circ} \mathrm{C}$. 
A equação da distribuição de temperatura para o modelo de chapa média, em variáveis adimensionais e em regime pseudoestacionário, extraída de Grong (1997), é definida pela Eq. (10):

$$
\frac{\theta}{n_{3}}=\exp (-\xi)\left[\sum_{i=-\infty}^{i=+\infty}\left(1 / \sigma_{i}\right) \exp \left(-\sigma_{i}\right)\right]
$$

e,

$$
\sigma_{i}=v R_{i} / 2 \alpha=\sqrt{\xi^{2}+\psi^{2}+(\zeta-2 i \delta)^{2}}
$$

Estudo de caso 3: Soldagem de aço baixa liga, considerando as mesmas condições e as mesmas temperaturas estudadas do caso 1. Os resultados apresentados na Fig. (9) mostram o mesmo comportamento do erro estudado no caso 1, com ligeira elevação de seus valores.
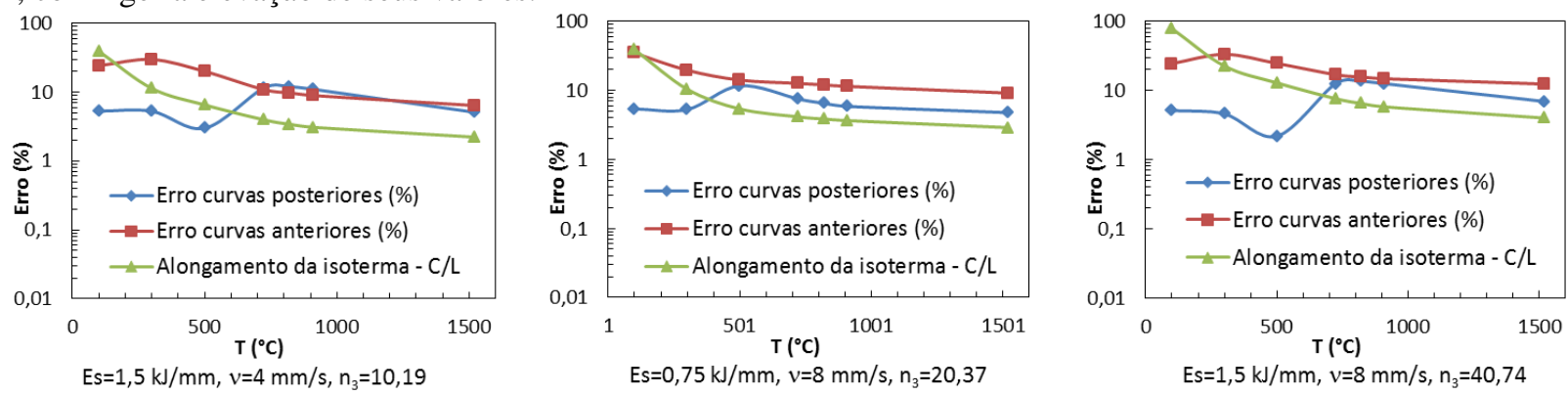

Figura 9. Curvas de erro para aço baixa liga, para a condição de transferência de calor para chapa média.

Estudo de caso 4: Soldagem de alumínio: processo GMAW, $\eta=0,8, I=260 \mathrm{~A}, U=25 \mathrm{~V}, v_{1}=3 \mathrm{~mm} / \mathrm{s}$ e $v_{2}=6 \mathrm{~mm} / \mathrm{s}$, espessura de $12 \mathrm{~mm}$. O erro aumentou em relação ao estudo de caso 2, embora os parâmetros de soldagem sejam os mesmos. Neste caso, o modelo leva em consideração a espessura da chapa. $\mathrm{O}$ ajuste entre as isotermas e a dupla elipse deteriorou em relação caso anterior, com erro máximo de $20,07 \%$ à temperatura de $40^{\circ} \mathrm{C}$, conforme valores na Fig. (10).
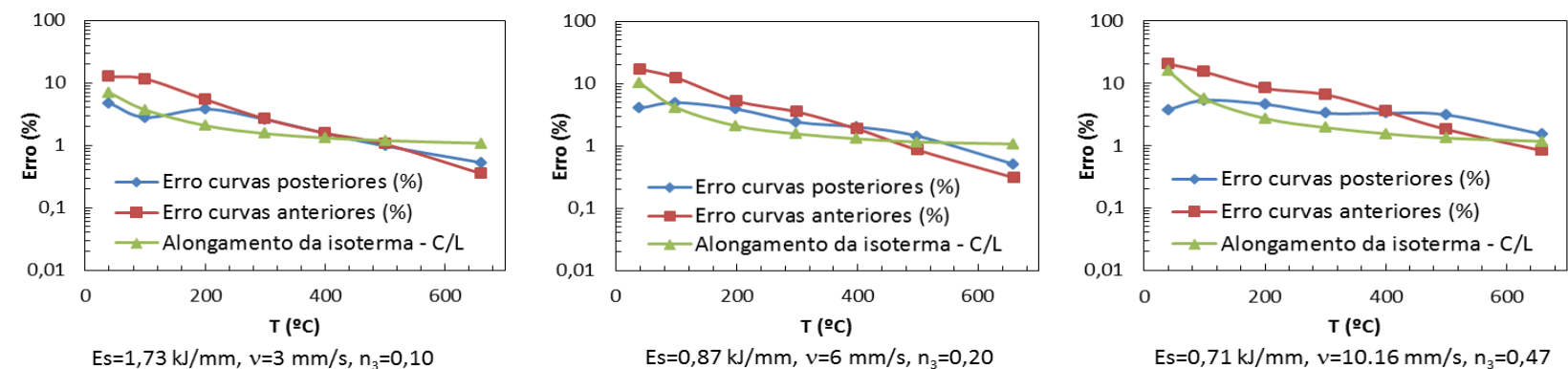

Figura 10. Curvas de erro para alumínio, para a condição de transferência de calor para chapa média.

\section{c) Estudo do erro em relação à chapa fina, em variáveis adimensionais}

A equação da distribuição de temperatura para o modelo de chapa fina, em variáveis adimensionais e em regime pseudoestacionário, extraída de Grong (1997), é definida pela Eq. (12) e pela Eq. (13):

$$
\begin{aligned}
& \theta \delta / n_{3}=\exp (-\xi) K_{0}\left(\sigma_{5}\right) \\
& \sigma_{5}=v r / 2 \alpha=\sqrt{\xi^{2}+\psi^{2}}
\end{aligned}
$$

Estudo de caso 5: Soldagem de aço baixa liga, espessura de $2 \mathrm{~mm}$, processo SMAW, $\eta=0,8, I=80 \mathrm{~A}, U=25 \mathrm{~V}$, $v_{1}=5 \mathrm{~mm} / \mathrm{s}$ e $v_{2}=10 \mathrm{~mm} / \mathrm{s}$. Dados obtidos do exemplo 1.11 de Grong (1997). Os resultados exibidos na Fig. (11) mostram um erro próximo a $5 \%$ entre a isoterma e a elipse posterior para todas as condições de soldagem. Porém mostra um erro máximo de $25 \%$ considerando a elipse anterior 

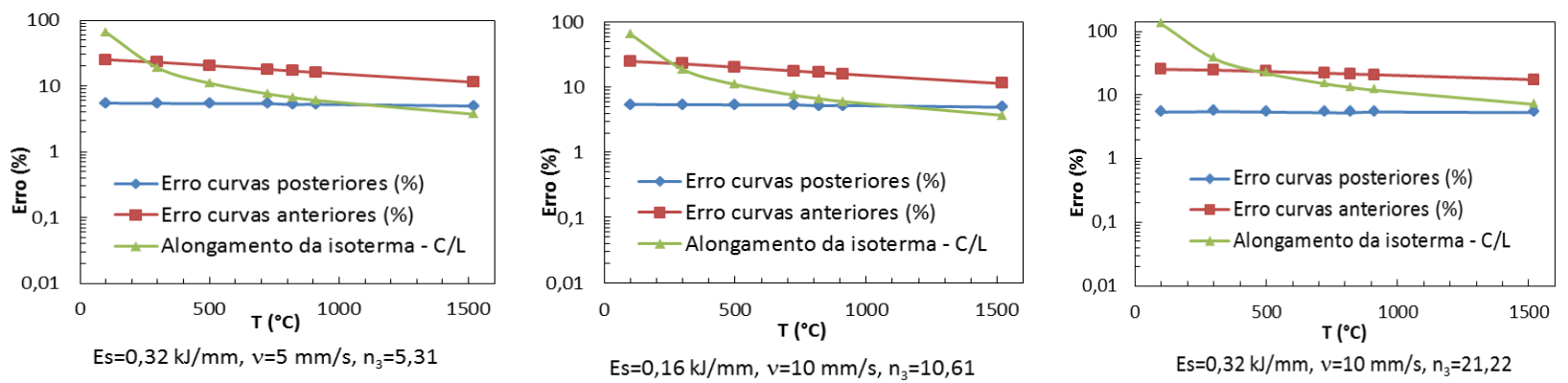

Figura 11. Curvas de erro para aço baixa liga, para a condição de transferência de calor para chapa fina.

Estudo de caso 6: Soldagem de alumínio: processo GTAW, $\eta=0,6, I=110 \mathrm{~A}, U=15 \mathrm{~V}, v_{1}=4 \mathrm{~mm} / \mathrm{s}$ e $v_{2}=8 \mathrm{~mm} / \mathrm{s}$, Dados obtidos do exemplo 1.10 de Grong (1997). A Fig. (12) mostra um erro mais significativo em relação à elipse anterior, mais especificamente quando $n_{3}=0,10$, onde o erro máximo é de aproximadamente $25 \%$.

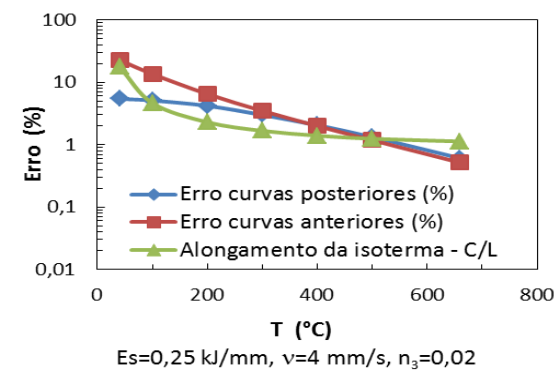

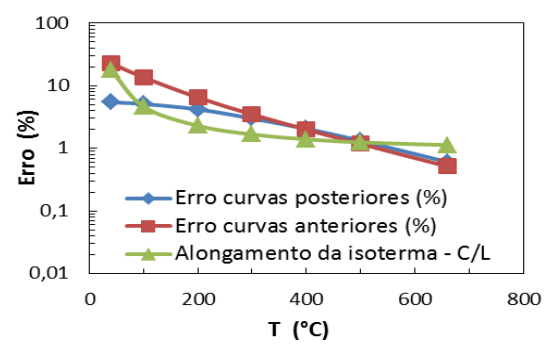

$E s=0,12 \mathrm{~kJ} / \mathrm{mm}, v=8 \mathrm{~mm} / \mathrm{s}, \mathrm{n}_{3}=0,05$

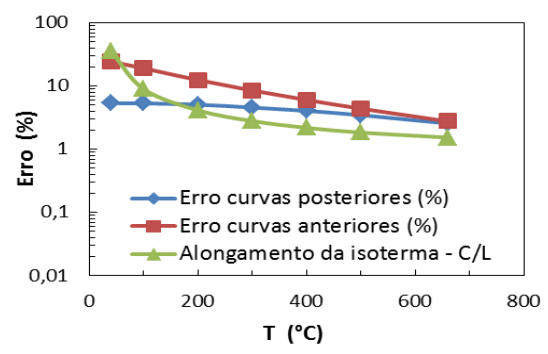

$E s=0,25 \mathrm{~kJ} / \mathrm{mm}, v=8 \mathrm{~mm} / \mathrm{s}, \mathrm{n}_{3}=0,10$

Figura 12. Curvas de erro para alumínio puro, condição de transferência de calor para chapa fina.

\section{4 - Simulação de soldagem de materiais fictícios pelo modelo chapa média}

Para verificar a influência do parâmetro operacional $n_{3}$ sobre os erros calculados foram simuladas soldagens de diversos materiais fictícios, onde a difusividade térmica $\alpha$ variou, enquanto as demais propriedades térmicas $\left(\rho c, H_{c} \mathrm{e}\right.$ $T_{c}$ ), e os parâmetros operacionais ( $\eta, v, T_{0}, U$ e $I$ ) mantiveram-se constantes. Nesta simulação foi utilizada uma energia de soldagem de $1,5 \mathrm{~kJ} / \mathrm{mm}$, e os erros foram medidos à temperatura de $500^{\circ} \mathrm{C}$, pelo modelo de placa média.

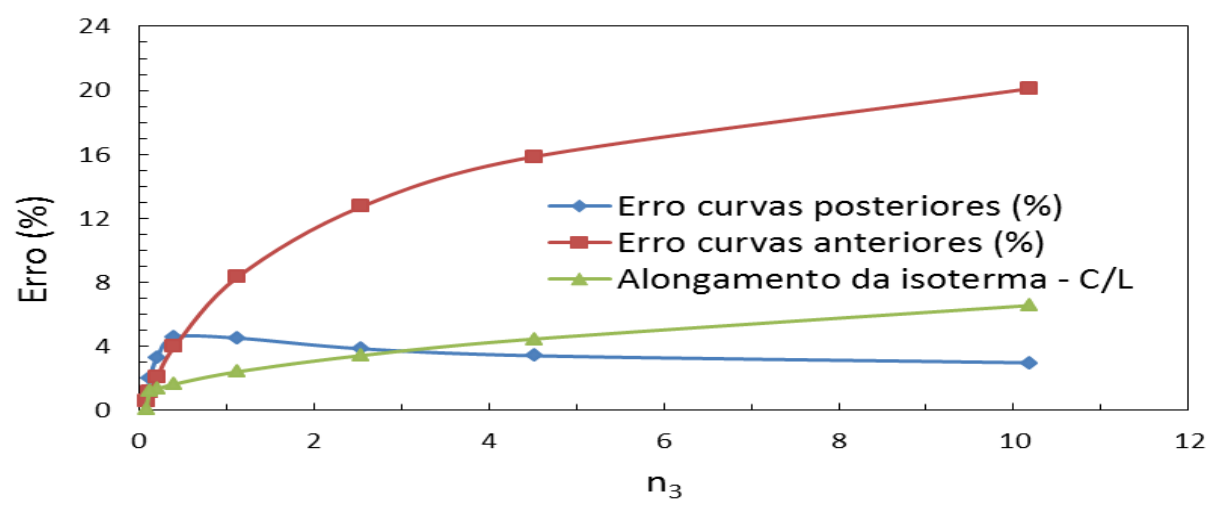

Figura 13. Variação do erro em função de $\mathbf{n}_{3}$ para o modelo de chapa média, $\mathbf{T}=\mathbf{5 0 0}{ }^{\circ} \mathrm{C}$.

Como se pode observar na Fig.(13), e em todos os casos estudados, o erro aumentou com o valor de $n_{3}$ e com o alongamento da isoterma $(\mathrm{C} / \mathrm{L})$. Ou seja, quanto mais alongada a isoterma, mais seu perfil se afasta do perfil de uma dupla elipse. A difusividade térmica chamou atenção em um primeiro momento, pois seu valor varia muito de um material para outro, como são os casos do alumínio e do aço. Entretanto, a velocidade de soldagem também influenciou nos resultados, e, consequentemente, a energia de soldagem.

\section{CONCLUSÕES}

O modelo de chapa grossa promove uma distribuição de calor em todas as direções, pois as isotermas resultantes do processo de soldagem não atingem a face oposta à da fonte de calor. Uma difusividade térmica alta, como é o caso do 
alumínio, tende a promover gradientes de temperatura mais homogêneos. Estes fatores, aplicado à soldagem de alumínio, gerou um erro máximo inferior a $5 \%$, na temperatura de $40^{\circ} \mathrm{C}$, mostrando boa conformidade entre a dupla elipse e a isoterma. Neste caso, a razão entre comprimento e largura da isoterma esteve bem próxima da unidade. Isto significa que as isotermas aproximam-se de circunferências, que são casos particulares de elipses.

Por outro lado, o erro aumentou para os outros modelos de chapa, tanto para soldagem de alumínio, quanto para soldagem de aço. Entretanto, em todas as situações simuladas, aquelas que indicaram menores erros foram as que produziram menores valores de $n_{3}$. Portanto, este pode ser o parâmetro utilizado para avaliar a eficiência da dupla elipse, pois ele engloba velocidade de soldagem, difusividade térmica e energia de soldagem, que também foram utilizadas para verificar o comportamento do erro.

Deve-se ressaltar que a decisão pela escolha da dupla elipse, não passa apenas pelo cálculo de baixo valor de $n_{3}$. Uma vez estabelecido o erro aceitável, torna-se ainda necessário verificar para qual temperatura de interesse o valor de $n_{3}$ atende, pois o erro também varia em função da temperatura. Na maioria dos casos estudados, as elipses anteriores à fonte de calor apresentaram os maiores erros. Isto ocorre porque os maiores gradientes de temperatura estão à frente da fonte de calor, como se pode observar na Fig. (4). Ou seja, tanto as isotermas quanto as elipses pouco se afastam no eixo das abcissas, mas se afastam mais no eixo das ordenadas, aumentando o alongamento das isotermas anteriores.

Embora, a definição de geometrias mais simples auxilie a simulação numérica, é preciso testar outras formas geométricas que produzam menos erros, baseando-se nos modelos de Rosenthal (1941).

Como relatado anteriormente, os estudos foram realizados considerando-se apenas as isotermas e duplas elipses calculadas nas faces superiores das chapas. Estendendo este estudo para o interior delas, o erro tende a ser maior ainda. Ou seja, a confrontação da geometria do duplo elipsoide em relação ao modelo de chapa grossa, é uma mera extensão da análise realizada em relação à dupla elipse, pois, assim como o duplo elipsoide é obtido pela rotação da dupla elipse, as isotermas abaixo da superfície superior da chapa grossa são também obtidas pela rotação das isotermas da face superior. Sendo assim, os erros calculados para este caso serão os mesmos.

Por outro lado, se a análise anterior for referida à chapa média, então os erros crescerão ainda mais, pois as isotermas abaixo da superfície superior desta chapa se degeneram das primeiras geometrias, à medida que temperaturas inferiores ao ponto de fusão são analisadas no interior da chapa, como se comprova pelos perfis das isotermas exibidos na Fig. (4).

\section{AGRADECIMENTOS}

Os autores gostariam de agradecer ao Programa de Pós-Graduação em Engenharia Metalúrgica e de Materiais da USP - Universidade de São Paulo, a CAPES - Coordenação de Aperfeiçoamento de Pessoal de Nível Superior, IFES Instituto Federal de Educação, Ciência e Tecnologia do Espírito Santo e FAPEAM - Fundação de Ampara à Pesquisa do Estado do Amazonas, pelo apoio financeiro.

\section{REFERÊNCIAS}

American Society for Metals, 1991. ASM Handbook: Heat Treating. USA: ASM International, 2173 p.

American Welding Society, 2001. Welding Handbook. 9. Edição: Cynthia L. Jenney e Annette O'Brien. Vol. 1. Miami, Florida.

Bate, S. K., Charles,R., Warren, A., 2009. Finite element analysis of a single bead-on-plate specimen using SYSWELD. International Journal of Pressure Vessels and Piping, 86, 73-78.

Boulton, N. S., Martin, H. E. L., 1937. Temperature Distribution During Welding. A Review of the Literature. Welding Journal, $16(9), 4-10$.

Goldak, J., Chakravarti, A., Bibby, M., 1984. A new finite element model for welding sources. Metallurgical Transactions B, 15, 299-305.

Grong, O., 1997. Metallurgical Modelling of Welding.Trondhein, Norway: Ed. The University Press Cambridge, 677 p.

Guimarães, P. B., Moura, M. L. M., Silva, R. J. F., Oliveira, C. A. N., Fernandes, L. E., Nascimento, A. C. S., Barbosa, J. M. A., Ferreira, R. A. S., 2012. Obtenção numérica dos ciclos térmicos de soldagem do aço inoxidável AISI 304. VII Congresso Nacional de Engenharia Mecânica, vol. 1, São Luis, Brasil.

Lancaster, J. F., 1999. Metallurgy of welding. Cambridge, Inglaterra: Ed. Abington Publishing, 468 p.

Rosenthal, D., 1935. Etude theorique du régime thermique pendant la soudure à la arc. Congrés National des Sciences, Compters Rendus, vol. 2, pp. (1277-1292). Bruxelas, Bélgica.

Rosenthal, D., Schmerber, H., 1938. Thermal Study of Arc Welding. Experimental Verification of Theoretical Formulas. Welding Journal, 17 (4), 2-8.

Rosenthal, D. 1941. Mathematical Theory of Heat Distribution during Welding and Cutting. Welding Journal, 20(5), 220-234.

Wainer, E., Brandi, S. D., Mello, F. D. H., 1992. Soldagem: Processo e Metalurgia. São Paulo, Brasil: Ed. Edgard Blücher Ltda. 494 p.

\section{RESPONSABILIDADE AUTORAL}

Os autores são os únicos responsáveis pelo conteúdo do material impresso incluído neste trabalho. 


\title{
EVALUATION OF THE GEOMETRY OF CURVES USED TO REPRESENT THE ISOTHERMS OF WELDED PLATE SURFACES
}

\author{
Dario Magno Batista Ferreira, dariomagno@ usp.br ${ }^{1}$ \\ Antônio do Nascimento Silva Alves, ansa@ usp.br ${ }^{1}$ \\ Rubelmar Maia de Azevedo Cruz Neto, rubelmar.neto@usp.br 1 \\ Sérgio Duarte Brandi, sbrandi@usp.br ${ }^{1}$
}

${ }^{1}$ EPUSP - Escola politécnica da USP, Dep. de Eng. Metalúrgica e de Materiais. Av. Professor Mello Moraes, 2463, CEP 05508-030, São Paulo - SP

\begin{abstract}
The heat transfer in welding is a phenomenon governed by Fourier equation, and occurs between the weld pool, its neighborhood and boundary conditions involved. It is characterized by high thermal gradients and high heating and cooling rates, which are observed by isothermal distribution and thermal cycles obtained. The isotherms are extremely important as they graphically describe the temperature gradients of heat transfer in all directions. An isotherm of particular interest is the melting temperature, for delimiting the contour of the weld pool, that is, separates the regions of liquid and solid metal. The models most commonly used in the literature allow describing temperature gradients in transient state. Among the models in literature, those developed by Rosenthal for heat transfer by conduction in welding processes in pseudo steady state is emphasized. The solutions of these models combine transcendental functions and special functions such as Bessel modified, depending on the boundary conditions and initial conditions for the model under study. They combine operational welding parameters, physical and geometrical properties of the plate to be welded. To obtain a graph of isotherms is necessary to use complex calculation using inverse functions of the solutions or by a process of calculating the roots of functions, for example. The mechanism will be adopted here. However, in numerical simulations of welding, authors have simplified one isotherm by a pair of ellipsoids. This brings the advantage of computational gain due to its simplicity compared to the Rosenthal model. In this sense, it is proposed to assess the extent of this gain to verify if this simplification is viable for different materials and welding operating conditions. This analysis was done to compare the geometry deviation of the ellipsoid with respect to the analytical solutions. The thermal diffusivity and welding speed were determinant in quantifying the error.
\end{abstract}

Key-Words: welding, simulation, isotherm, thermal cycles, ellipsoid 